\title{
Combinação do ADMM com Homogeneização Matemática na Modelagem da Dispersão de Poluentes na Atmosfera
}

\author{
Camila Pinto da $\operatorname{Costa}^{1}$ (iD, Leslie Darien Pérez-Fernández ${ }^{1}$ (D), Karine Rui ${ }^{2}$ id, \\ Julián Bravo-Castillero ${ }^{3}$ id \\ 1Departamento de Matemática e Estatística, Instituto de Física e Matemática, \\ Universidade Federal de Pelotas, Pelotas, RS, Brasil. \\ ${ }^{2}$ Programa de Pós-Graduação em Engenharia Mecânica, \\ Universidade Federal do Rio Grande do Sul, Porto Alegre, RS, Brasil. \\ ${ }^{3}$ Departamento de Matemática y Mecánica, \\ Instituto de Investigaciones en Matemáticas Aplicadas y en Sistemas, Universidad Nacional \\ Autónoma de México, México, DF, México.
}

Recebido em 7 de Fevereiro de 2017 - Aceito em 23 de Julho de 2017

\begin{abstract}
Resumo
O método multicamadas de advecção-difusão (ADMM) produz soluções semianalíticas precisas dos problemas de valores de contorno/iniciais para equações de advecção-difusão com coeficientes variáveis que modelam a dispersão de poluentes na atmosfera, e apresenta o menor custo computacional quando comparado com outros métodos baseados em transformadas integrais. Contudo, em situações operativas tais como desastres naturais/industriais que resultam na fuga de poluentes na atmosfera, é necessário aferir rapidamente e com exatidão a distribuição da concentração dos poluentes no nível do solo para minimizar o impacto na saúde e na economia. Aqui, para acelerar a disponibilidade de resultados com mínima perda de precisão, o ADMM é combinado com homogeneização matemática, cujo emprego na modelagem de dispersão de poluentes parece ser novidade. A abordagem proposta é comparada com a aplicação direta do ADMM e às observações do experimento de Hanford para avaliar a exatidão das estimativas, assim como sua eficiência computacional, considerando condições atmosféricas estáveis e a influência da velocidade de deposição. Os resultados mostram que a combinação do ADMM com a homogeneização matemática apresenta uma redução significativa no custo computacional com pouca perda de precisão.
\end{abstract}

Palavras-chave: modelagem da dispersão de poluentes na atmosfera, método multicamadas de advecção-difusão, homogeneização matemática.

\section{Combining the ADMM and Mathematical Homogenization for Atmospherical Pollutant Dispersion Modeling}

\begin{abstract}
The advection-diffusion multilayer method (ADMM) produces accurate semi-analytical solutions of initial/boundaryvalue problems for advection-diffusion equations with variable coefficients that model pollutant dispersion in the atmosphere, and exhibits lower computational cost in comparison to other integral transform-based methods. However, in operative situations such as natural/industrial disasters resulting in the release of pollutants to the atmosphere, it is necessary to assess rapidly and accurately the ground-level distribution of pollutant concentration in order to minimize the impact on health and economy. Here, in order to accelerate the availability of results with little loss of accuracy, the ADMM is combined with mathematical homogenization, whose use in pollutant dispersion modeling seems to be new. The proposed approach is compared with the direct application of the ADMM and to the observations of the Hanford experiment in order to access both its accuracy and computational cost, for stable atmospheric conditions and considering the influence of deposition velocity. The results show that the combination of the ADMM and mathematical homogeni-
\end{abstract}

Autor de Correspondência: Camila Pinto da Costa, camila.costa@ufpel.edu.br. 
zation reduces remarkably the computation cost with little loss of accuracy.

Keywords: modeling pollutant dispersion in the atmosphere, advection-diffusion multilayer method, mathematical homogenization.

\section{Introdução}

O método multicamadas de advecção-difusão (ADMM, siglas em inglês - ver Costa et al., 2006) é um dos métodos baseados em transformadas integrais utilizados para resolver analiticamente os problemas de valores de contorno/iniciais para equações de advecção-difusão com coeficientes variáveis que modelam a dispersão de poluentes na atmosfera. Foi relatado em Moreira et al. (2010) que o ADMM produz estimativas da concentração de poluentes tão exatas quanto as produzidas mediante outros métodos baseados em transformadas integrais tais como o GILTT (siglas em inglês para técnica da transformada integral de Laplace generalizada - ver Moreira et al., 2009), mas com custo computacional significativamente menor. Esta última característica é essencial em situações operativas tais como em desastres naturais/industriais nas quais estimativas precisas e rápidas (idealmente em tempo real) da distribuição e concentração a nível do solo dos poluentes liberados na atmosfera. Assim, a disponibilidade de estimativas rápidas e precisas em condições operativas é necessária para a prevenção e diminuição do impacto de emergência de poluição tanto na saúde quanto na economia.

O ADMM considera a aproximação constante por partes dos coeficientes contínuos do perfil de velocidade do vento e da difusividade turbulenta na direção vertical $z \in[0, h](h$ é a altura da camada limite planetária (CLP)), e a aplicação da transformada de Laplace. Tal aproximação é a média local dos coeficientes variáveis em cada subcamada. Dessa forma, o problema original com coeficientes contínuos é aproximado por um problema com coeficientes constantes por partes que produzirá resultados mais precisos e com maior custo computacional a medida que a discretização da CLP for mais fina.

Neste trabalho, para acelerar a disponibilidade de resultados com a menor perda de exatidão possível, o ADMM é combinado com homogeneização matemática (Bensoussan et al., 1978; Pobedrya, 1984; Bakhvalov e Panasenko, 1989). De forma resumida, a homogeneização de meios heterogêneos baseia-se nas hipóteses de separação de escalas estruturais e do contínuo. Isto é, a estrutura do meio (aqui, a CLP) é caracterizada por um parâmetro geométrico pequeno $\varepsilon$ definido como o quociente dos comprimentos característicos de ambas as escalas microscópica e macroscópica, de maneira que propriedades efetivas ou macroscópicas podem lhe ser atribuídas ao meio heterogêneo (sempre que as condições de contorno sejam uniformes). Com tais considerações, a hipótese de homogeneidade equivalente é válida, ou seja, existe um meio homogêneo ideal que é equivalente ao meio heterogêneo no sentido de que as propriedades físicas do primeiro são as propriedades efetivas do segundo. Do ponto de vista matemático, a hipótese de homogeneidade equivalente é válida se a solução do problema original (aqui, do ADMM) com coeficientes variáveis (aqui, constantes por partes), que modela o meio heterogêneo (aqui, a CLP), converge para a solução do chamado problema homogeneizado com coeficientes constantes, que modela o meio homogêneo associado, na norma do espaço em que tais soluções são procuradas quando $\varepsilon \rightarrow 0^{+}$. Em outras palavras, a norma da diferença de tais soluções é de ordem de uma potência de $\varepsilon$. Aqui, os coeficientes efetivos da difusividade turbulenta vertical e do perfil da velocidade média do vento são obtidos a partir do problema do ADMM no espaço de Laplace para condições estáveis. Segundo nosso melhor conhecimento, a aplicação da homogeneização matemática à modelagem da dispersão de poluentes na atmosfera parece ser uma novidade na área. Adicionalmente, a velocidade de deposição seca no solo é considerada.

Este trabalho está organizado como segue: a seção 2 contém a formulação dos problemas originais e do ADMM; a seção 3 descreve os processos de solução via o ADMM na sua forma pura e em combinação com a homogeneização matemática; resultados de várias simulações são apresentados e discutidos na seção 4; e as conclusões finais são declaradas na seção 5 .

\section{Formulação dos Problemas}

Considere o sistema de coordenadas cartesiano $O_{X Y Z}$ tal que a fonte de poluentes está localizada no ponto $(0,0$, $H_{s}$ ) e a direção do vento é paralela ao eixo $x$. Nesta abordagem inicial da homogeneização matemática na modelagem da dispersão de poluentes, considera-se o modelo estacionário bidimensional obtido da equação de advecção-difusão de Arya (1995) ao integrar lateralmente e desprezar a difusão longitudinal. Ainda, considera-se o fenômeno da deposição seca no solo.

\subsection{Problema original}

O modelo descrito acima é formulado como segue:

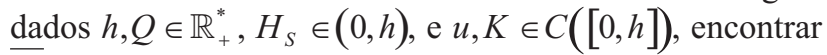
$\overline{c^{y}} \in \mathcal{H}^{1}\left(\mathbb{R}_{+} \times[0, h]\right)$ tal que

$$
\begin{aligned}
& u(z) \frac{\partial \overline{c^{y}}}{\partial x}=\frac{\partial}{\partial z}\left[K(z) \frac{\partial c^{y}}{\partial z}\right], x \in \mathbb{R}_{+}^{*}, z \in(0, h), \\
& \left.K(0) \frac{\partial \overline{c^{y}}}{\partial z}\right|_{z=0}=V_{d} \overline{c^{y}}(x, 0), x \in \mathbb{R}_{+}^{*},
\end{aligned}
$$




$$
\begin{aligned}
& \left.K(h) \frac{\partial \overline{c^{y}}}{\partial z}\right|_{z=h}=0, x \in \mathbb{R}_{+}^{*}, \\
& u(z) \overline{c^{y}}(0, z)=Q \delta\left(z-H_{s}\right), z \in(0, h),
\end{aligned}
$$

em que $u(z)$ é a velocidade média do vento, $K(z)$ é a difusão turbulenta, $\overline{c^{y}}(x, z)$ é a concentração média de poluentes integrada lateralmente, $C(\cdot)$ é o espaço das funções contínuas, $\mathcal{H}^{1}(\cdot)$ é o espaço das funções de quadrado integrável cujas derivadas de primeira ordem são de quadrado integrável, $Q$ é a taxa de emissão da fonte poluidora, $V_{d}$ é a velocidade de deposição seca no solo, e $\delta(\cdot)$ é a função delta de Dirac. Para simplificar a notação, escreve-se $c(x, z)=\overline{c^{y}}(x, z)$. Neste trabalho, consideram-se as seguintes parametrizações de $K(z)$ e $u(z)$ de Hanna (1982) e Panofsky e Dutton (1984), respectivamente:

$$
K(z)=\tau_{L_{w}} \sigma_{w}^{2},
$$

em que

$$
\tau_{L_{w}}=0.1 \frac{h}{\sigma_{w}}\left(\frac{z}{h}\right)^{\frac{4}{5}},
$$

é a escala de tempo de decorrelação vertical,

$$
\sigma_{w}=1.3 u_{*}\left(1-\frac{z}{h}\right)
$$

é a variação da velocidade vertical turbulenta, $u *$ é a velocidade de atrito, e

$$
u(z)=u_{1}\left(\frac{z}{z_{1}}\right)^{p},
$$

em que $u_{1}$ é a velocidade do vento na altura $z=z_{1}$, e $p$ está relacionado à intensidade da turbulência (Irwin, 1979). Ainda, considera-se que a velocidade de deposição $V_{d}$ é dada por (Hanna, 1982; Arya, 1999)

$$
V_{d}(z)=0.01 u(z) \text {. }
$$

\subsection{Problema ADMM}

Seja $\left\{z_{n}\right\}_{n=\overline{0, N}} \subset[0, h], N \in \mathbb{N}$, uma partição da CLP. Em cada subcamada $\left(z_{n-1}, z_{n}\right), n=\overline{1, N}$, de espessura $\Delta z_{n}=z_{n}-z_{n-1}$ da CLP, considere os valores médios de $K(z)$ e $u(z)$ :

$$
A_{n}=\frac{1}{\Delta z_{n}} \int_{z_{n-1}}^{z_{n}} A(z) d z, A=K, u,
$$

o que define aproximações constantes por partes de $K(z)$ e $u(z)$ dadas por $A(z) \approx \underline{A}(z)=A_{n}, z \in\left(z_{n-1}, z_{n}\right), n=\overline{1, N}$, $A=K, u$. Seja $c_{n}(x, z)=c(x, z),(x, z) \in \mathbb{R}_{+}^{*} \times\left(z_{n-1}, z_{n}\right)$, $n=\overline{1, N}$. Assim, o problema original Eqs. (1)-(4) é aproximado pelo problema

$$
\begin{aligned}
& u_{n} \frac{\partial c_{n}}{\partial x}=K_{n} \frac{\partial^{2} c_{n}}{\partial z^{2}}, z \in\left(z_{n-1}, z_{n}\right), n=\overline{1, N}, \\
& c_{n}\left(x, z_{n}\right)=c_{n+1}\left(x, z_{n}\right), n=\overline{1, N-1}, \\
& \left.K_{n} \frac{\partial c_{n}}{\partial z}\right|_{z=z_{n}}=\left.K_{n+1} \frac{\partial c_{n+1}}{\partial z}\right|_{z=z_{n}}, n=\overline{1, N-1}, \\
& \left.\frac{\partial c_{1}}{\partial z}\right|_{z=0}=\frac{V_{d}}{K_{1}} c_{1}(x, 0), \\
& \left.\frac{\partial c_{N}}{\partial z}\right|_{z=h}=0, \\
& c_{n}(0, z)=\frac{Q}{u_{n}} \delta\left(z-H_{s}\right) \delta_{n n}, z \in\left(z_{n-1}, z_{n}\right), n=\overline{1, N},
\end{aligned}
$$

em que Eq. (12) e (13) impõem a continuidade nos pontos da partição $z=z_{n}, n=\overline{1, N-1}$, à concentração de poluentes $\mathrm{e}$ ao fluxo turbulento, respectivamente. Ainda, $\delta_{n n}$ na condição de contorno Eq. (16) é a delta de $\operatorname{Kronecker}\left(\delta_{n \bar{n}}=1 \mathrm{se}\right.$ $n=\bar{n}, \delta_{n \bar{n}}=0$ se $n \neq \bar{n}$ ), em que $n=\bar{n}$ indica a subcamada da CLP em que a fonte poluidora está localizada, ou seja, $H_{S} \in\left(z_{\bar{n}-1}, z_{-}\right)$.

\section{Estratégias de Resolução}

\subsection{Solução ADMM}

De aplicar a transformada de Laplace $\mathcal{L}[\cdot]$ em relação à variável $x$ ao problema ADMM Eqs. (11)-(16), segue que, para cada $s \in \mathbb{C}$,

$$
\begin{gathered}
\frac{d^{2} \varsigma_{n}}{d z^{2}}-\frac{u_{n} s}{K_{n}} \varsigma_{n}(s, z)=-\frac{Q}{K_{n}} \delta\left(z-H_{s}\right) \delta_{n \bar{n}}, z \in\left(z_{n-1}, z_{n}\right), n=\overline{1, N}, \\
\varsigma_{n}\left(s, z_{n}\right)=\varsigma_{n+1}\left(s, z_{n}\right), n=\overline{1, N-1}, \\
\left.K_{n} \frac{d \zeta_{n}}{d z}\right|_{z=z_{n}}=\left.K_{n+1} \frac{d \varsigma_{n+1}}{d z}\right|_{z=z_{n}}, n=\overline{1, N-1}, \\
\left.\frac{d \zeta_{1}}{d z}\right|_{z=0}=\frac{V_{d}}{K_{1}} \varsigma_{1}(s, 0), \\
\left.\frac{d \varsigma_{N}}{d z}\right|_{z=h}=0
\end{gathered}
$$

em que $\varsigma_{n}(s, z)=\mathcal{L}\left[c_{n}(x, z)\right]$ Logo, para $z \in\left(z_{n-1}, z_{n}\right)$, $n=\overline{1, N}$, a solução do problema ADMM no espaço de Laplace dado por Eqs. (17)-(21) é

$$
\begin{aligned}
& \varsigma_{n}(s, z)=A_{n} \exp \left\{R_{n}(s) z\right\}+B_{n} \exp \left\{-R_{n}(s) z\right\} \\
& -\frac{Q \operatorname{senh}\left\{R_{n}(s)\left(z-H_{s}\right)\right\}}{K_{n} R_{n}(s)} H\left(z-H_{s}\right) \delta_{n n},
\end{aligned}
$$

em que $R_{n}(s)=\sqrt{u_{n} s / K_{n}}$ e $H(\cdot)$ é a função de Heaviside. Os coeficientes constantes $A_{n}$ e $B_{n}$ em Eq. (22) são obtidos 
resolvendo o sistema de equações algébricas lineares que resulta de substituir Eq. (22) nas condições Eqs. (18)-(21).

Logo, a solução do problema ADMM Eqs. (11)-(16) segue de aplicar a transformada de Laplace inversa $\mathcal{L}^{-1}[\cdot]$ à Eq. (22). Note que a complexidade da solução Eq. (22) requer inverter numericamente a transformada de Laplace, de maneira que a solução final é semianalítica. O algoritmo de inversão mais utilizado é a quadratura de Gauss, mas aqui utiliza-se o algoritmo Fixed-Talbot (Abate e Valkó, 2004) que é computacionalmente mais robusto (também, ver, Moreira et al., 2014). Portanto, para $z \in\left(z_{n-1}, z_{n}\right)$, $n=\overline{1, N}$, a solução $c_{n}(x, z)=\mathcal{L}^{-1}\left[\varsigma_{n}(s, z)\right]$ do problema ADMM Eqs. (11)-(16) é

$c_{n}(x, z)=\frac{r}{M}\left[\frac{1}{2} \varsigma_{n}(r, z) \exp \{r x\}\right.$

$\left.+\sum_{k=1}^{M-1} \operatorname{Re}\left\{\exp \left\{x S\left(\theta_{k}\right)\right\} \varsigma_{n}\left(S\left(\theta_{k}\right), z\right)\left(1+i \omega\left(\theta_{k}\right)\right)\right\}\right]$,

em que $r \in \mathbb{R}_{+}^{*}$ é fixo, $i=\sqrt{-1}$, e $S\left(\theta_{k}\right)=r \theta_{k}\left(\cot \theta_{k}+i\right)$, $\omega\left(\theta_{k}\right)=\theta_{k}+\left(\theta_{k} \cot \theta_{k}-1\right) \cot \theta$, e $\theta_{k}=k \pi / M \in(-\pi, \pi)$ (para mais detalhes, ver, Abate e Valkó, 2004).

\subsection{Aproximação via homogeneização matemática}

O problema homogeneizado do problema ADMM no espaço de Laplace Eqs. (17)-(21) é

$$
\begin{aligned}
& \frac{d^{2} \bar{\zeta}}{d z^{2}}-\frac{\hat{u} s}{\hat{K}} \bar{\zeta}(s, z)=-\frac{Q}{\hat{K}} \delta\left(z-H_{S}\right), \quad z \in(0, h), \\
& \left.\frac{d \bar{\zeta}}{d z}\right|_{z=0}=\frac{V_{d}}{\hat{K}} \bar{\zeta}(s, 0), \\
& \left.\frac{d \bar{\zeta}}{d z}\right|_{z=h}=0
\end{aligned}
$$

em que $\bar{\zeta}(s, z)$ é tanto o valor médio de $\varsigma(s, z)$ quanto a transformada de Laplace da aproximação $\bar{c}(x, z)$ obtida via homogeneização matemática da solução do problema ADMM (11)-(16), $\bar{\zeta}(s, z)=\mathcal{L}[\bar{c}(x, z)]$, e os coeficientes efetivos $\hat{u}$ e $\hat{K}$ são aproximados pelas médias aritmética e harmônica, respectivamente, das aproximações constantes por partes de $u(z)$ e $K(z)$, ou seja,

$$
\hat{u}=\frac{1}{h} \int_{0}^{h} u(z) d z \approx \frac{1}{h} \int_{0}^{h} \underline{u}(z) d z=\frac{1}{h} \sum_{n=1}^{N} \Delta z_{n} u_{n},
$$

$\mathrm{e}$

$$
\hat{K}^{-1}=\frac{1}{h} \int_{0}^{h} \frac{d z}{K(z)} \approx \frac{1}{h} \int_{0}^{h} \frac{d z}{\underline{K}(z)}=\frac{1}{h} \sum_{n=1}^{N} \frac{\Delta z_{n}}{K_{n}}
$$

Para justificar matematicamente a formulação do problema homogeneizado Eqs. (24)-(28), observe que, para cada $s \in \mathbb{C}$ e cada $N \in \mathbb{N}$, o problema ADMM no espaço de Laplace Eqs. (17)-(21) pode ser escrito como

$$
\begin{aligned}
& -\frac{d}{d z}\left[\underline{K}(z) \frac{d \zeta}{d z}\right]+\underline{u}(z) s \zeta(s, z) \\
& =Q \delta\left(z-H_{S}\right), z \in(0, h) \backslash\left\{z_{n}\right\}, \quad n=\overline{1, N-1}, \\
& {[|\varsigma(s, z)|]_{z=z_{n}}=0, \quad n=\overline{1, N-1},} \\
& {\left[\left|\underline{K}(z) \frac{d \zeta}{d z}\right|\right]_{z=z_{n}}=0, \quad n=\overline{1, N-1},} \\
& \left.\underline{K}(0) \frac{d \zeta}{d z}\right|_{z=0}=V_{d \zeta}(s, 0), \\
& \left.\underline{K}(h) \frac{d \zeta}{d z}\right|_{z=h}=0,
\end{aligned}
$$

em que $[\mid \cdot]_{z=z_{n}}$ denota o operador de salto nos pontos de descontinuidade $z=z_{n}$ de $\underline{K}(z)$ e $\underline{u}(z)$, e as derivadas são consideradas no sentido generalizado (ver, por exemplo, o Capítulo 1 de Bakhvalov e Panasenko, 1989). Este problema tem uma solução generalizada $\varsigma(s, z)=\varsigma(s, z)$ cuja existência pode ser provada mediante um princípio do máximo (por exemplo, o Lema 1.1, página 95, de Pao, 1992). Tomando o parâmetro geométrico que descreve a separação de escalas como $\varepsilon=1 / N$, tem-se que $N \rightarrow \infty$ é equivalente a $\varepsilon \rightarrow 0^{+}$, isto é, o limite no qual se baseia a homogeneização matemática. De aplicar o Lema 4.1, p. 63, de Tartar (2009), segue que se existem $\hat{K}, \hat{u} \in \mathbb{R}_{+}^{*}$ e $\bar{\varsigma} \in \mathcal{H}^{1}(\mathbb{C} \times[0, h])$ tais que $\underline{K}^{-1}(z) \rightarrow \hat{K}^{-1}, \underline{u}(z) \rightarrow \hat{u}$, e $\varsigma(s, z) \rightarrow \bar{\zeta}(s, z)$ quando $N \rightarrow \infty$, então, para cada $s \in \mathbb{C}$, $\overline{\bar{\zeta}}(s, z)$ é solução de

$$
-\hat{K} \frac{d^{2} \bar{\zeta}}{d z^{2}}+\hat{u} s \bar{\zeta}(s, z)=Q \delta\left(z-H_{S}\right), z \in(0, h),
$$

e $\underline{K}(z) d \underline{\varsigma} / d z \rightarrow \hat{K} d \bar{\zeta} / d z$ quando $N \rightarrow \infty$, o qual é aplicado para obter as condições de contorno Eqs. (25) e (26). Por outro lado, para o caso puramente difusivo, em Gorbachev e Pobedrya (1997) provou-se, mediante uma abordagem que envolve funções de Green e teoremas de valor médio, uma fórmula cuja versão unidimensional é $\hat{K}=\left\langle\underline{K}^{-1}(z)\right\rangle^{-1}$, em que $\langle\cdot\rangle=h^{-1} \int(\cdot) d z$ é o operador de valor médio, que coincide com a fórmula Eq. (28) para o coeficiente efetivo da difusividade turbulenta. Ainda, se o domínio é $\varepsilon$-periódico, o Teorema 2.6, página 33, de Cioranescu e Donato (1999) garante que $\underline{K}^{-1}(y) \rightarrow\left\langle\underline{K}^{-1}(y)\right\rangle_{Y}$ e $\underline{u}(y) \rightarrow\langle\underline{u}(y)\rangle_{Y}$ quando $N \rightarrow \infty$, com o operador de valor médio $\langle\cdot\rangle_{Y}=|Y|^{-1} \int_{Y}(\cdot) d y$ definido localmente sobre o período estrutural $Y$ э $y=z / \varepsilon$. Dado que, neste caso, o valor médio local coincide com o valor médio sobre todo o domínio, é possível identificar $\langle\cdot\rangle$ $\operatorname{com}\langle\cdot\rangle_{Y}$, o qual leva a tomar $\hat{K}=\left\langle\underline{K}^{-1}(z)\right\rangle^{-1}$ e $\hat{u}=\langle\underline{u}(z)\rangle^{-1}$, 
que no caso unidimensional se transformam nas fórmulas Eqs. (27) e (28) para os coeficientes efetivos.

Logo, sendo $\hat{R}(s)=\sqrt{\hat{u} s / \hat{K}}$, a solução do problema homogeneizado no espaço de Laplace definido por Eqs. (24)-(28) é

$$
\begin{aligned}
& \bar{\zeta}(s, z)=\frac{Q}{\hat{K} \hat{R}(s)} \frac{\hat{K} \hat{R}(s) \cosh \{\hat{R}(s) z\}+V_{d} \sinh \{\hat{R}(s) z\}}{\hat{K} \hat{R}(s) \sinh \{\hat{R}(s) z\}+V_{d} \cosh \{\hat{R}(s) z\}} \\
& \times \cosh \left\{\hat{R}(s)\left(h-H_{S}\right)\right\}-\frac{Q}{\hat{K} \hat{R}(s)} \sinh \left\{\hat{R}(s)\left(h-H_{S}\right)\right\} \\
& \times H\left(z-H_{S}\right)
\end{aligned}
$$

Dessa forma, a aproximação por homogeneização matemática da solução do problema ADMM Eqs. (11)-(16) segue de aplicar a transformada de Laplace inversa à Eq. (35). Como na seção anterior, devido à complexidade da solução Eq. (35), é necessário inverter numericamente a transformada de Laplace, de maneira que a aproximação final da solução é semianalítica, para o qual utilizamos o algoritmo Fixed-Talbot de Abate e Valkó (2004). Assim, a aproximação por homogeneização matemática da solução do problema ADMM Eqs. (11)-(16) é

$$
\begin{aligned}
& \bar{c}(x, z)=\frac{r}{M}\left[\frac{1}{2} \bar{\zeta}(r, z) \exp \{r x\}\right. \\
& \left.+\sum_{k=1}^{M-1} \operatorname{Re}\left\{\exp \left\{x S\left(\theta_{k}\right)\right\} \bar{\zeta}\left(S\left(\theta_{k}\right), z\right)\left(1+i \omega\left(\theta_{k}\right)\right)\right\}\right]
\end{aligned}
$$

\section{Resultados e Discussão}

Para avaliar o desempenho da abordagem proposta combinando o ADMM e homogeneização matemática dada por Eqs. (35) e (36), foram realizadas simulações computacionais cujos resultados foram comparados com os resultados da aplicação direta do ADMM dada por Eqs. (22) e (23), e com as observações do experimento de Hanford (Doran et al., 1984). Os parâmetros usados no algoritmo de inversão numérica da transformada de Laplace são $M=100$ e $r=2 M / 901 x$.

Este experimento foi realizado em Hanford, uma região semiárida no sudeste de Washington (EUA), no período de 18/05/1983 a 27/06/1983. A superfície do terreno é plana com vegetação de gramíneas de 1 a $2 \mathrm{~m}$ de altura de e comprimento de rugosidade de $z_{0}=0.03 \mathrm{~m}$. O experimento consistiu em liberar dois traçadores: um poluente que deposita, o sulfeto de zinco ( $\mathrm{ZnS}$ ) e outro que não deposita, o hexafluoreto de enxofre $\left(\mathrm{SF}_{6}\right)$, de uma torre com vazão de $Q=0.3 \mathrm{~g} / \mathrm{s}$ a uma altura de $H_{s}=2 \mathrm{~m}$. Foram conduzidos seis experimentos. Os dados foram coletados a $1.5 \mathrm{~m}$ do solo em cinco arcos de amostragem localizados a distâncias de 100 m, $200 \mathrm{~m}, 800 \mathrm{~m}, 1600 \mathrm{~m}$ e $3200 \mathrm{~m}$ a partir da fonte com distância angular de $8^{\circ}, 4^{\circ}, 4^{\circ}, 2^{\circ}$ e $3^{\circ}$, respectivamente. Uma torre meteorológica de $122 \mathrm{~m}$ foi instalada a $100 \mathrm{~m}$ ao norte da área de liberação com sensores de velocidade e direção do vento, e temperatura em altitudes de $2.1 \mathrm{~m}, 9.1$ $\mathrm{m} ; 5.2 \mathrm{~m}, 30.5 \mathrm{~m}, 61 \mathrm{~m}, 91 \mathrm{~m}$ e $122 \mathrm{~m}$ para monitorar as condições durante as liberações dos traçadores para garantir que o material fosse lançado durante períodos de condições de vento e de estabilidade adequadas. As liberações ocorreram durante condições de moderadamente estáveis a quase-neutras.

A Fig. 1 apresenta os perfis horizontais da concentração de poluentes próxima do nível do solo resultados das simulações computacionais de ambos os métodos em comparação com as observações do experimento de Hanford para $\mathrm{p}=0.35$ e $z_{1}=10 \mathrm{~m}$. Nas diversas simulações realizadas observou-se que a aplicação direta do ADMM Eqs. (22)-(23) consumiu de 15 a 20 vezes mais tempo computacional que a combinação do ADMM com a homogeneização matemática Eqs. (35)-(36). Este fato é relevante quando se lida com uma grande quantidade de dados. Por outro lado, observa-se na Fig. 1 que ambas as curvas têm o mesmo comportamento qualitativo, sendo que a abordagem proposta subestima (resp. superestima) os valores da concentração de poluentes em regiões próximas (resp. afastadas) da fonte poluidora. Mesmo assim, a abordagem proposta fornece aproximações da concentração em comparação com as observações experimentais.

Para avaliar a precisão dos resultados obtidos via a abordagem proposta em comparação com os resultados obtidos via o ADMM e as observações do experimento de Hanford, o desempenho de ambos os métodos é medido em termos dos índices estatísticos propostos em Hanna (1989), a saber, o erro quadrático médio normalizado (NMSE), o coeficiente de correlação $(C O R)$, o fator de dois $(F A 2)$, o viés fracionário $(F B)$, e o desvio padrão fracionário $(F S)$. Estes índices estatísticos são calculados como segue, em que a barra indica a média aritmética e $\sigma$ denota o desvio padrão:

$$
\begin{aligned}
& \text { NMSE }=\frac{\overline{\left(c_{p}-c_{o}\right)^{2}}}{\overline{c_{p} c_{o}}}, \\
& \text { COR }=\frac{\overline{\left(c_{o}-\bar{c}_{o}\right)\left(c_{p}-\bar{c}_{p}\right)}}{\overline{\sigma_{o} \sigma_{p}}}, \\
& F A 2=(\text { percentual de }) \frac{c_{p}}{c_{o}} \in[0.5,2] \\
& F B=\frac{\bar{c}_{o}-\bar{c}_{p}}{0.5\left(\bar{c}_{o}+\bar{c}_{p}\right)}, \\
& F S=\frac{\sigma_{o}-\sigma_{p}}{0.5\left(\sigma_{o}+\sigma_{p}\right)} .
\end{aligned}
$$

A Tabela 1 apresenta os valores dos índices estatísticos Eqs. (37)-(41) dos resultados das simulações computacionais apresentados na Fig. 1. Como esperado, ob- 

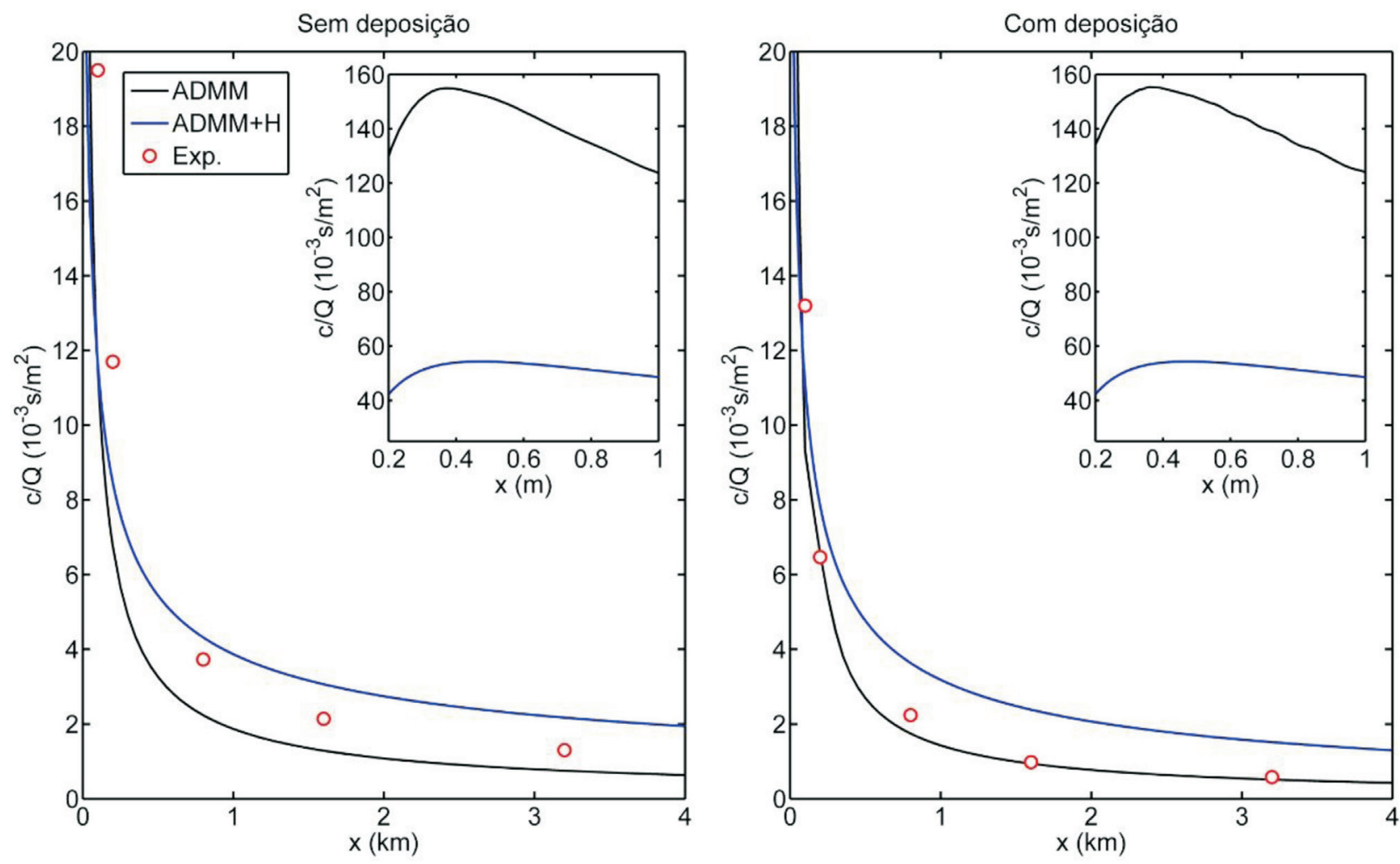

Figura 1 - Comparação dos resultados da solução ADMM (22)-(23) e a aproximação por homogeneização (35)-(36) das simulações computacionais junto com as observações do experimento de Hanford.

Tabela 1 - Índices estatísticos em comparação com o experimento de Hanford.

\begin{tabular}{lcccccc}
\hline Deposição & Método & NMSE & COR & FA2 & FB & FS \\
\hline Com & ADMM+H & 0,55 & 0,920 & 0,389 & $-0,635$ & $-0,370$ \\
deposição & ADMM & 0,09 & 0,903 & 1,000 & $-0,040$ & $-0,011$ \\
Sem & ADMM+H & 0,06 & 0,939 & 0,889 & $-0,134$ & 0,160 \\
deposição & ADMM & 0,12 & 0,866 & 0,722 & $-0,176$ & 0,035 \\
\hline
\end{tabular}

serva-se que a precisão da abordagem proposta é inferior à do ADMM, mas aceitável, sobre tudo quando se considera o ganho em tempo na disponibilização dos resultados, o qual é muito útil para avaliações iniciais em situações de emergência.

Tal perda de precisão pode ser superada considerando outros aspectos da homogeneização matemática. Com efeito, aqui considerou-se apenas a aproximação de ordem zero, isto é, a solução do problema homogeneizado. Seguindo a metodologia da homogeneização assintótica, é possível construir soluções assintóticas de qualquer ordem, o qual permite ter boas aproximações da solução exata do problema original com a precisão desejada.

\section{Conclusões}

Neste trabalho, para acelerar a disponibilidade de resultados com a menor perda de exatidão possível, apre- sentou-se a combinação do ADMM com homogeneização matemática. Segundo nosso melhor conhecimento, a aplicação da homogeneização matemática à modelagem da dispersão de poluentes na atmosfera parece ser uma novidade na área. Adicionalmente, a velocidade de deposição seca no solo foi considerada. Para avaliar o desempenho da abordagem proposta combinando o ADMM e homogeneização matemática, foram realizadas simulações computacionais cujos resultados foram comparados com os resultados da aplicação direta do ADMM e as observações do experimento de Hanford. Nas diversas simulações realizadas observou-se que a aplicação direta do ADMM, o qual apresenta o menor custo computacional quando comparado com outros métodos baseados em transformadas integrais, consumiu aproximadamente de 15 a 20 vezes mais tempo computacional que a combinação do ADMM com a homogeneização matemática. Este fato é relevante tanto quando se lida com uma grande quantidade de dados, quanto em avaliações iniciais em situações de emergência, o qual compensa a pequena perda de precisão observada.

\section{Agradecimentos}

Os autores agradecem o projeto CAPES No. 88881.030424/2013-01 pelo suporte financeiro. 


\section{Referências}

ABATE, J.; VALKÓ, P.P. Multi-precision Laplace transform inversion. International Journal for Numerical Methods in Engineering, v. 60, p. 979-993, 2004.

ARYA, S.P. Modeling and parameterization of near source diffusion in weak winds. Journal in Applied Meteorology, v. 34, p. 1112-1122, 1995.

ARYA, S.P. Air Pollution Meteorology and Dispersion. Oxford University Press, New York, 1999.

BAKHVALOV, N.; PANASENKO, G. Homogenisation: averaging processes in periodic media. Kluwer, Dordrecht, 1989.

BENSOUSSAN, A.; LIONS, J. L.; PAPANICALOU, G. Asymptotic analysis for periodic structures. North-Holland, Amsterdam, 1978.

CIORANECU, D.; DONATO, P. An Introduction to homogenization. Oxford University Press, Oxford, 1999.

COSTA, C.P.; VILHENA, M.T.; MOREIRA, D.M.; TIRABASSI, T. Semi-analytical solution of the steady three-dimensional advection-diffusion equation in the planetary boundary layer. Atmospheric Environment, v. 40, p. 5659-5669, 2006.

DORAN, J.C.; ABBEY, O.B.; BUCK, J.W.; GLOVER, D.W.; HORST, T.W.; LEE, R.N.; LLOYD, F.D. Field Validation of Exposure Assessment Models. United States: Environmental Protection Agency, Environmental Sciences Research Laboratory, 1984.

GORBACHEV, V.I.; POBEDRYA, B.E. The effective characteristics of inhomogeneous media. Journal of Applied Mathematics and Mechanics, v. 61, p. 145-151, 1997.

HANNA, S.R. Applications in air pollution modeling. In: Nieuwstadt, F.T.M., Van Dop, H. (Eds). Atmospheric Turbulence and Air Pollution Modelling. Dordrecht Reidel Publishing, p. 275-310, 1982.

HANNA, S.R. Confidence limits for air quality model evaluations, as estimated by bootstrap and jackknife resampling methods. Atmospheric Environment, v. 23, p. 1385-1398, 1989.

IRWIN, J.S. A theoretical variation of the wind profile power-law exponent as a function of surface roughness and stability. Atmospheric Environment, v. 13, p. 191-194, 1979.

MOREIRA, D.M.; MORAES, A.C.; GOULART, A.G.; ALBUQUERQUE, T.T.A. A contribution to solve the atmospheric diffusion equation with eddy diffusivity depending on source distance. Atmospheric Environment, v. 83, p. 254-259, 2014.

MOREIRA, D.M.; VILHENA, M.T.; BUSKE, D.; TIRABASSI, T. The state-of-art of the GILTT method to simulate pollutant dispersion in the atmosphere. Atmospheric Research, v. 92, p. 1-17, 2009.

MOREIRA, D.M.; VILHENA, M.T.; TIRABASSI, T.; BUSKE, D.; COSTA, C.P. Comparison between analytical models to simulate pollutant dispersion in the atmosphere. International Journal of Environment and Waste Management, v. 6, p. 327-344, 2010.

PANOFSKY, H.A.; DUTTON, J.A. Atmospheric turbulence: models and methods for engineering applications. John Wiley \& Sons, New York, 1984.

PAO, C.V. Nonlinear parabolic and elliptic equations. Plenum, New York, 1992.

PAULSON, C.A. The mathematical representation of wind and temperature profiles in the unstable atmospheric surface layer. Journal of Applied Meteorology, v. 9, p. 857-861, 1970.

POBEDRYA, B.E. Mechanics of composite materials. Moscow State University Press, Moscow (in Russian), 1984.

TARTAR, L. The general theory of homogenization: a personalized introduction. Springer-Verlag, Berlin Heidelberg, 2009.

This is an Open Access article distributed under the terms of the Creative Commons Attribution Non-Commercial License which permits unrestricted non-commercial use, distribution, and reproduction in any medium provided the original work is properly cited. 